\title{
What Is New With Omicron Variant of SARS-CoV-2 in Children?
}

\author{
Stefan Bittmann ${ }^{\mathrm{a}, \mathrm{b}}$, Elisabeth Luchter ${ }^{\mathrm{a}}$, Elena Moschuring-Alieva ${ }^{\mathrm{a}}$, \\ Lara Bittmann ${ }^{\mathrm{a}}$, Gloria Villalon ${ }^{\mathrm{a}}$
}

\section{To the Editor}

The World Health Organization (WHO) has classified the risk of severe acute respiratory syndrome coronavirus 2 (SARS-CoV-2) variant B.1.1.529 as very high since November 29. Omicron is designated as of concern [1-9]. This classification, according to the WHO definition, is a signal that a variant may be more contagious or lead to more severe disease [1-9]. In addition, "variants of concern" risk makes conventional vaccines, drugs, or coronavirus measures less effective $[1,6,8,9]$. The WHO had only classified the Alpha, Beta, Gamma and Delta variants as worrisome. The WHO warns of the emergence of even more dangerous virus variants. The Omicron variant is massively on the rise [1-9]. At the moment, all indications are that it is even more infectious than the previously dominant, already highly contagious Delta variant. Vaccine manufacturers also believe that two doses will not provide complete protection against infection with Omicron, but will still provide protection against severe disease $[1,6,8,9]$. For the best possible protection, a third booster vaccination is mandatory. But it is not only in terms of infectivity and vaccine protection that Omicron is said to differ from Delta and the previous variants, the symptoms are also said to be somewhat different. Omicron affects all age groups, including the youngest. Since mid-November, the Robert Koch Institute (RKI) has recorded more than 44,600 infections with the coronavirus variant among $0-14$ years old children (as of January 21, 2022). Seven-day incidence has now reached new record levels. The indicator, which describes the number of infections within 7 days per 100,000 inhabitants, is currently highest in the 5 - 14 years age group. It was to be expected that there would also be an increase in infections among children and adolescents. The previous variants, Alpha and Delta, did not differentiate between ages.

Manuscript submitted January 26, 2022, accepted February 8, 2022

Published online February 24, 2022

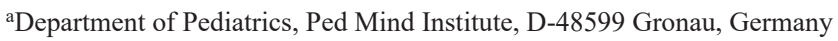
${ }^{b}$ Corresponding Author: Stefan Bittmann, Medical and Finance Center Epe, Ped Mind Institute (PMI), D-48599 Gronau-Epe, Germany.

Email: stefanbittmann@gmx.de

doi: https://doi.org/10.14740/jocmr4671
Because Omicron is even more easily transmissible, the virus variant can spread more quickly in the population than its predecessors [1-9]. This means that the risk of it hitting younger people increases. The number of hospitalized young children in the USA who tested positive for the coronavirus rose last week to its highest level since the start of the pandemic. That is according to data released Friday by the US Centers for Disease Control (CDC), the American equivalent of Germany's RKI. The number is alarming; however, the experts give the first all-clear with regard to the severity of the disease progression after an Omicron infection. The CDC data showed that about four in every 100,000 children aged 4 or younger were infected with coronavirus when they were hospitalized on January 1 . The rate doubled from the previous month and tripled from the same time of last year. This shows the extent to which Omicron is spreading among even the youngest, leading to very high case rates in this age group. The increase in Omicron cases in the USA affects all populations. In slightly older children, however, there was no change in the Omicron wave: the hospitalization rate for infected children aged 5 - 11 years remained about the same at 0.6 per 100,000 . One explanation for the increased number of cases in the younger age group could be that many children are currently coming to hospitals with other respiratory illnesses; in other words, they are being admitted with coronavirus rather than because of it. Older children in the USA especially are often already vaccinated. CDC data from the USA, where more than 8.6 million vaccinations were given to 5 - 11 years old children, also showed very good tolerability of the coronavirus vaccine in the age group. There were found few cases of myocarditis. The risk was 10 times lower than in 12 - 15 years old children. There is, to date, no increased risk in girls. Pediatric inflammatory multisystem syndrome (PIMS) is an inflammatory reaction that has increased in children since the coronavirus pandemic. It can occur 3 to 4 weeks after infection. It affects 3 out of 10,000 infected children, 1.6 of whom require treatment in intensive care units. But the near-certainty that children are immune to severe courses of the disease is beginning to crack. The number of children infected with the Omicron virus variant is rising in some countries, and so is the number of those among them who need to be treated in hospital. In the USA, for example, in 1 week, from December 21 to December 28, the number of children with coronavirus disease 2019 (COV- 
ID-19) who were hospitalized increased by $66 \%$, to an average of 378 children per day. And in the UK, 226 children between the ages of 6 and 17 years were hospitalized with coronavirus during the week of December 19 to 26. A total of 286 under 5 years old British children were also hospitalized during the same period. In German hospitals, there are currently a relatively large number of younger children with proven Omicron infections. This is the result of a new overview published by the RKI on Monday, January 3 . The numbers collected in it goes back to November 15 of last year. In it, the RKI recorded a total of 632 Omicron cases in 0 - 4 years old children in those roughly 1.5 months. Of these children, 12 were treated in hospital. Twelve does not sound like a lot at first, but it is about $1.9 \%$ of all infected 0 - 4 years old children. The next age group, whose situation the RKI surveyed, consists of both adolescents and young adults: Of a total of 13,731 15 - 34 years old cases infected with Omicron, 91 required hospital treatment. That represents about $0.66 \%$. However, the survey does not indicate how many of these individuals were under 18 years. Particularly in the age group of 0 - 4 years, these data are potentially cause for concern because children under 5 years of age are not yet allowed to be vaccinated against COVID-19. But do the RKI figures really mean that children are particularly at risk from Omicron? Not necessarily, said experts from the German Society for Child and Adolescent Medicine (DGKJ) and the German Society for Pediatric Infectiology (DGPI). Detailed analyses, which they said in a joint statement from the DGKJ and DGPI, put the fears into perspective. "It is still too early to make a definitive assessment, but experiences from the Tshwane District in South Africa, London and Australia are reassuring," it adds. It also says that previous pandemic waves, in which, for example, the Alpha and then the Delta variants spread rapidly, did not pose a particularly severe risk to children. Coincidence diagnoses play a large role with the risen numbers. This means that children who were admitted to hospital for a completely different reason were then tested positive for the Omicron variant, and were therefore included in the statistics by chance.

\section{Acknowledgments}

None to declare.

\section{Financial Disclosure}

None to declare.

\section{Conflict of Interest}

None to declare.

\section{Informed Consent}

Not applicable.

\section{Author Contributions}

SB wrote the article and did all the literature research. EL and EM did the proof reading. LB did the English translation. GV proofread the manuscript towards reference list.

\section{Data Availability}

The data supporting the findings of this study are available from the corresponding author upon reasonable request.

\section{References}

1. Ledford H. How severe are Omicron infections? Nature. 2021;600(7890):577-578.

2. Garcia-Beltran WF, St. Denis KJ, Hoelzemer A, Lam EC, Nitido AD, Sheehan ML, Berrios C, et al. mRNAbased COVID-19 vaccine boosters induce neutralizing immunity against SARS-CoV-2 Omicron variant. Cell. 2022;185(3):457-466.e4.

3. Ludvigsson JF. Convulsions in children with COVID-19 during the Omicron wave. Acta Paediatr. 2022.

4. Dejnirattisai W, Huo J, Zhou D, Zahradnik J, Supasa P, Liu C, Duyvesteyn HME, et al. SARS-CoV-2 OmicronB.1.1.529 leads to widespread escape from neutralizing antibody responses. Cell. 2022;185(3):467-484.e415.

5. Wang L, Berger NA, Kaelber DC, Davis PB, Volkow ND, $\mathrm{Xu}$ R. COVID infection severity in children under 5 years old before and after Omicron emergence in the US. medRxiv. 2022.

6. Viana R, Moyo S, Amoako DG, Tegally H, Scheepers C, Althaus CL, Anyaneji UJ, et al. Rapid epidemic expansion of the SARS-CoV-2 Omicron variant in southern Africa. Nature. 2022.

7. Chen LL, Chua GT, Lu L, Chan BP, Wong JS, Chow CC, $\mathrm{Yu} \mathrm{TC}$, et al. Omicron variant susceptibility to neutralizing antibodies induced in children by natural SARSCoV-2 infection or COVID-19 vaccine. Emerg Microbes Infect. 2022;11(1):543-547.

8. Girard B, Tomassini JE, Deng W, Maglinao M, Zhou H, Figueroa A, Ghamloush SS, et al. mRNA-1273 Vaccineelicited Neutralization of SARS-CoV-2 Omicron in Adolescents and Children. medRxiv. 2022.

9. Tian D, Sun Y, Xu H, Ye Q. The emergence and epidemic characteristics of the highly mutated SARS-CoV-2 Omicron variant. J Med Virol. 2022. 